\section{$\underset{\substack{\text { hommes } \\ \text { \& migrations }}}{ }$}

\section{Hommes \& migrations}

Revue française de référence sur les dynamiques

migratoires

1306 | 2014

Ecriture et migration

\title{
La présence arabo-musulmane en Languedoc et en Provence à l'époque médiévale
}

\section{Marc Terrisse}

\section{OpenEdition \\ Journals}

Édition électronique

URL : http://journals.openedition.org/hommesmigrations/2837

DOI : 10.4000/hommesmigrations.2837

ISSN : 2262-3353

Éditeur

Musée national de l'histoire de l'immigration

Édition imprimée

Date de publication : 1 avril 2014

Pagination : 126-128

ISBN : 978-2-919040-27-8

ISSN : $1142-852 X$

\section{Référence électronique}

Marc Terrisse, "La présence arabo-musulmane en Languedoc et en Provence à l'époque médiévale », Hommes \& migrations [En ligne], 1306 | 2014, mis en ligne le 31 juillet 2014, consulté le 22 septembre 2020. URL : http://journals.openedition.org/hommesmigrations/2837 ; DOI : https://doi.org/10.4000/ hommesmigrations.2837 


\section{LA PRÉSENCE ARABO-MUSULMANE EN LANGUEDOC ET EN PROVENCE À L'ÉPOQUE MÉDIÉVALE}

MARC TERRISSE, président et fondateur de l'association Le Regard de l'Autre

$\mathrm{L}^{2}$ 'association Le Regard de l'Autre a été créée en 2012 pour travailler sur la présence de la civilisation arabo-musulmane en France de la période médiévale jusqu'à la fin de l'époque moderne. L’association est partie du constat que les interactions liées aux migrations, aux échanges culturels et économiques entre la France et la zone culturelle arabo-musulmane n'étaient pas ou peu connues du grand public en raison de travaux scientifiques assez épars et peu diffusés pour les deux périodes concernées.

\section{La réalisation d'une exposition itinérante}

En 2013, Le Regard de l'Autre a organisé l'exposition itinérante Nos ancêtres les Sarrasins, présentant les dernières découvertes archéologiques d'époque médiévale réalisées dans les régions méditerranéennes françaises et illustrant une présence arabo-musulmane ou des échanges avec le monde islamique. Cette présence n'est certes pas aussi spectaculaire que dans la péninsule Ibérique ou l'Italie du Sud. Néanmoins, plusieurs éléments permettent de reconstituer ce passé à l'aide de nombreuses hypothèses.

Une exposition repose sur la transformation d'un discours scientifique érudit et parfois contradictoire en un média de communication accessible aux néophytes mais intéressant également pour les passionnés d'histoire. Pour répondreà ces impératifs, un comité scientifique composé d'archéolo- gues, d'historiens et de conservateurs de musée a été mis sur pied. Ce comité était coordonné par un muséographe en mesure de compiler l'ensemble des travaux scientifiques et de recourir aux médias de communication adéquats pour toucher un large public. Retenir uniquement les régions méridionales du Languedoc et de la Provence comme objet de l'exposition est rapidement apparu comme une évidence. Un certain nombre de travaux historiques leur ont été consacrés et des trouvailles archéologiques plutôt récentes sont venues étayer ces recherches. Un inventaire de l'ensemble de ces travaux, du matériel archéologique découvert et des objets présents dans les musées a ensuite été réalisé de façon à dégager les thématiques principales faciles à illustrer. Cette étape a consisté à contacter un grand nombre d'institutions œuvrant dans la science archéologique ou dans la conservation du patrimoine, principalement des musées. Cet inventaire fondé sur de nombreuses lectures, sur l'identification de sources archéologiques, iconographiques et sur la sélection de ce corpus a pris un an.

Cinq thèmes ont ensuite été sélectionnés à l'aide du comité scientifique pour l'exposition. Dans leur très grande majorité, ces thèmes devaient pouvoir s'appuyer sur des photos d'objets conservés dans des musées. Voici les thématiques présentées dans le cadre de l'exposition : les événements militaires liés à la conquête arabo-musulmane dans le sud de la Gaule et la démystification de la bataille de Poitiers ; la présence arabo-musulmane en Languedoc au VIIIle siècle et en Provence 
au Xe siècle ; les échanges commerciaux entre les ports méditerranéens comme Marseille, Agde, Montpellier et l'aire culturelle arabo-musulmane aux XII-XIII siècles ; la présence de communautés musulmanes à Marseille et à Montpellier pendant cette période ; enfin, la transmission de la culture arabo-andalouse en Languedoc et en Provence par le biais des juifs séfarades au XIIe siècle. Une douzaine de panneaux ont servi de supports à l'exposition pour qu'elle soit facilement transportable. C'est un texte court et précis s'appuyant sur des cartes, des images provenant d'objets conservés dans des musées et dont il a fallu acquérir les droits de diffusion, qui a animé le scénario de cette exposition. L'esthétique des panneaux a été travaillée à travers des dessins ou des peintures exécutés par un artiste et illustrant certains thèmes comme les batailles ou les échanges commerciaux. L'exposition se veut délibérément non encyclopédique car il ne s'agit pas de créer un livre à ciel ouvert. L'objectif est de fournir les informations essentielles tout en exposant les différentes hypothèses liées aux découvertes archéologiques ou aux interprétations historiques. Beaucoup de thématiques font l'objet de questions relevant le caractère complexe du sujet. Ainsi, les visiteurs peuvent s'approprier cette thématique peu diffusée et auront le loisir d'aller chercher des informations supplémentaires.

En partie financée par la Mairie de Paris, l'exposition itinérante a été hébergée par certaines mairies d'arrondissement et des discussions avec des institutions comme l'Institut du monde arabe, le Musée d'histoire de Marseille, l'Institut français du Maroc, plusieurs établissements scolaires sont actuellement menées pour l'accueillir. Les réactions du public ont été recueillies grâce à un livre d'or. Celui-ci est globalement positif. Beaucoup de visiteurs ignoraient tout de ce passé et de cette présence. D’autres disent qu'ils le connais- saient mais essentiellement par le biais d'une certaine forme de "mythologie" liée à l'imaginaire sur la présence sarrasine dans le sud de la France. La majorité ignoraient la probable présence de communautés musulmanes à Marseille ou Montpellier au Moyen Âge.

\section{La présence arabo-berbère en Languedoc et en Provence du VIII ${ }^{e}$ au $\mathrm{X}^{\mathrm{e}}$ siècle}

Les troupes arabo-berbères venant d'Espagne se sont établies dans le Languedoc pendant une partie du VIII e siècle. Des sources arabes mentionnent Carcassonne, Nîmes, mais c'est surtout à Narbonne, Arbûnah en Arabe, que les troupes se fixent pendant quarante ans environ, soit de 719 à 759'. Des sceaux mentionnant "Arbûnah", découverts sur le site de Ruscino situé à proximité de Perpignan, prouvent cette occupation². Des fals et des dinars arabes ont été retrouvés dans plusieurs localités languedociennes 3 . Même après la bataille de Sigean en 737, remportée par les Francs sur les Arabo-Berbères, il semble que ces derniers soient restés autour de Narbonne et dans la région de Perpignan, qu'ils quittent probablement encore plus tardivement, peut-être vers 785 . Cela démonte également le mythe de la bataille de Poitiers, qui aurait "chassé" tous les musulmans d'une France qui n'existait pas encore.

En Provence, quatre épaves du $X^{e}$ siècle découvertes lors de fouilles sous-marines entre Marseille et Cannes contenaient des jarres, des armes, des céramiques et divers objets du quotidien. L'architecture de la coque de ces bateaux est typique du monde islamique. Les sources arabes et chrétiennes mentionnent de leur côté l'existence d'un établissement peuplé d'Arabo-Berbères dans l'arrière-pays tropézien à la Garde-Freinet au

1. Philippe Sénac, "Présence musulmane en Languedoc, réalités et vestiges", in Cahier de Fanjeaux, $\mathrm{n}^{\circ}$ 18, "Islam et chrétiens du Midi", Toulouse, Privat, 2000, pp. 43-57. 2. Rémy Marichal, Philippe Sénac, "Sceaux arabes, Ruscino et la conquête musulmane”, in L’Archéologue, $n^{\circ}$ 112, Fév-Mars 2011, pp. 49-51. 3. Jean Duplessy, "La circulation des monnaies arabes en Europe occidentale du VIII au XIII siècle", in Revue numismatique, Paris, 1956. 


\section{INITIATIVES}

$X^{e}$ siècle $e^{4}$. Les fouilles archéologiques menées sur place n'ont à ce jour rien donné. Les bateaux pourraient donc soit faire office de cargaisons commerciales se dirigeant vers les ports, soit conforter l'existence de raids sarrasins mentionnés dans les sources écrites de l'époque 5 .

\section{Commerce international et présence de communautés musulmanes}

Aux XII-XIII siècles, Marseille, Montpellier, Narbonne ou Agde créent des entrepôts à Tunis, Ceuta, Tanger, Oran, Alexandrie ou Tyr. Les vases à décoration islamique datant des XIII-XIVe siècles découverts dans l'Hérault et exposés au musée de l'Éphèbe d'Agde illustrent cette activité commerciale. À Marseille, un four de technologie islamique datant du XII e siècle a été découvert dans le quartier des potiers. Il permettait de produire in situ des céramiques de style islamique. La question est de savoir comment ce transfert de technologie a été réalisé6. Or une tombe de caractère islamique a été mise au jour ainsi que des inscriptions en arabe?. Tous ces éléments concourent à créditer l'hypothèse de la présence d'une communauté musulmane dans la ville à cette époque. On peut faire le même constat à Montpellier, où des tombes à écriture koufique du XIIe siècle ont été exhumées. Elles sont à mettre en parallèle avec des sources écrites laissant penser qu'il y avait une communauté musulmane à Montpellier au Moyen Âge 8 .

\section{Les transferts culturels à l'époque médiévale}

Les connaissances du monde arabo-musulman en matière de médecine ou de philosophie nous sont parvenues le plus souvent par l'intermédiaire des juifs de culture arabe installés en Andalousie ou au Maghreb et venus s'établir en Languedoc et en Provence. C'est le cas de la dynastie des Ibn Tibbon venue à Montpellier au XII ${ }^{e}$ siècle pour fuir les persécutions de la dynastie almohade'. Les Ibn Tibbon vont traduire de l'arabe vers I'hébreu Averroès et d'autres érudits. Ils introduisent de ce fait la pensée arabo-andalouse en dehors de la sphère culturelle islamique pour nourrir les milieux intellectuels des villes languedociennes et provençales.

Cette exposition apparaît comme la première étape d'un projet plus vaste visant à actualiser et à enrichir ces connaissances. Des problématiques liées aux mots d'origine arabe en français ou en occitan ${ }^{10}$, à l'influence des poèmes arabes sur les troubadours ${ }^{11}$, aux jeux comme les échecs ${ }^{12}$ ou aux transferts gastronomiques ${ }^{13}$ mériteraient d'être explicitées. II en va de même pour l'époque moderne avec l'histoire des Morisques, prochain grand chantier de l'association.

4. Philippe Sénac, "Note sur le Fraxinet des Maures", in Annales du Sud-Est varois, t. XV, 1990, pp. 19-23. 5. Yan Codou, Germain Butaud, "Dans la gueule des Sarrasins. Le monastère de Saint-Honorat de Lérins et les incursions sarrasines VIIIe-XIIe siècles : histoire et archéologie", colloque “Héritages arabo-islamiques dans l'Europe méditerranéenne, archéologie, histoire, anthropologie”, Marseille, septembre 2013, http://www.inrap.fr/archeologie-preventive/Ressources/Heritages-arabo-islamiques/p-16050-Conference-par-Germain-Butaud-et-YannCodou.htm. 6. Henri Marchesi, Jacques Thiriot, Lucy Vallauri, “Le bourg médiéval des potiers : un échange culturel en Méditerranée”, in Archeologia, n² 290, 1993, pp. 29-30. 7. Marc Bouiron, Philippe Mellinand, Quand les archéologues redécouvrent Marseille, Paris, Gallimard, 2013, pp. 122-124. 8. Jacques Jomier, “Note sur les stèles funéraires arabes de Montpellier”, in Cahier de Fanjeaux, n 18, Privat, Toulouse, 2000, pp. 62-63. 9. Paul Fenton, "Le rôle des traducteurs juifs dans la transmission du savoir arabe", in Histoire de l'Islam et des musulmans en France, Paris, Albin Michel, 2006, pp. 240-241. 10. Peter Ricketts, "L'influence de la culture arabe sur le lexique de l'ancien occitan”, in Dominique Billy, François Clément, Annie Combes (dir.), L'Espace lyrique méditerranéen au Moyen Âge, Toulouse, Presses universitaires du Mirail, 2006, pp. 291-295. 11. Rachel Arié, “Ibn Hazm et l'amour courtois”, in Revue de l'Occident musulman et de la Méditerranée, Aix-enProvence, 1985, p. 76. 12. Voir Matthieu Grandet, Catalogue exposition "Échecs et trictrac", 23 juin-18 novembre 2012, Château de Mayenne, Arles, Actes Sud, 2012. 13. Jean-François Kosta-Théfaine, Le Viandier. Recettes de cuisine du Moyen Âge, Clermont-Ferrand, Paleo, 2011. 